\title{
Square Water as a Solvent: Monte Carlo Simulations
}

\author{
Nara Guisoni* and Vera Bohomoletz Henriques ${ }^{\dagger}$ \\ Instituto de Física, Universidade de São Paulo, \\ C.P. 66318, сер 05315-970, São Paulo, SP, Brazil
}

Received on 15 October, 2000

\begin{abstract}
Square water takes into account the directionality of hydrogen bonds. The model is reviewed and its properties as a solvent for apolar particles are studied through Monte Carlo simulations. Specific heat measurements are used to identify phase separation. Data for comparison with the lattice gas on the square lattice are presented and the relation to non-associating solvents is discussed. Data for the frequency of hydrogen bonds as a function of temperature indicate a slower rate of bond breaking for the hydration shell as compared to bulk water particles.
\end{abstract}

\section{Introduction}

Apolar molecules are poorly soluble in water $[1,2]$. It has been known for a long time that this is due, in many cases, not to enthalpic, but to entropic effects: the dissolution in water of such molecules results in a decrease both in enthalpy (which would lead to large solubility) and in entropy, despite the disruption of the net of hydrogen bonds characteristic of water. This effect has been qualitatively explained quite some time ago [1] in terms of an effect known as hydrophobic hydration: a tendency of the water molecules to "strengthen" their structure around the apolar molecules. Hydrophobic interaction, leading to a tendency toward aggregation of a nonpolar solute, would also result. NMR and other experimental evidence $[3,4]$ is in favour of the hydration hypothesis. Molecular dynamics simulations of model water solutions with atomic detail have probed these ideas, but evidence is often contradictory [5] and still not quite conclusive as to which microscopic properties are responsible for the lower entropy [6-9]. The simulation of molecular models with atomic detail is restricted, by current computer facilities, to short times. Simplified models are therefore an alternative tool. In a recently proposed very simple lattice model, Barkema and Widom[10] impose ordering on dissolution and analyse the effective solute interaction. In this paper we follow the more usual approach: we adopt a lattice model for the solvent and look for signs of induced structure around apolar solutes.

The hydrogen bond network[11] is thought to be responsible for other special properties of pure water such as a maximum in the density curve as a function of temperature. Above the melting temperature the tetrahedrally coordinated open ice structure is distorted, retaining most of the HB network but accomodating some broken bonds and increasing slightly the number of nearest neighbours, and thus the density [12]. At higher temperatures, usual expansion takes place, under increasing disruption of the net [12]. Consideration of water with atomic detail has led to the development of a number of different models [13, 14], all of which present some sort of discrepancy in relation to experimental thermodynamic or structural properties of liquid water. On the other hand, numerous simplified statistical models [15] have been proposed in the search for the main features which may explain the behaviour exhibited by this special liquid. Square water is one of these models, whose properties were studied by Nadler and Krausche[16]. Due probably to its simple thermodynamic behaviour, in which no phase transition is present, it is scarcely mentioned in the literature as a model for water ${ }^{1}$.

Square ice incorporates the hydrogen bond net but does not allow for distortions or density fluctuations. Thus the model is incapable of presenting anything like a liquid-ice transition. However, it could represent the net and its fluctuations at temperatures characteristic of liquid water. Rotations which lead to disruption of a bond are considered in an extreme (discrete) form. We have undertaken to study the effect of such an associated model solvent [18] on apolar solutes, both in

\footnotetext{
*Electronic address: nara@fge.if.usp.br

†Eletronic address: vhenriques@if.usp.br

${ }^{1}$ Precisely the same model has been used recently to describe "spin-ice" behaviour of frustrated ferromagnets[17], but the resemblance to Nadler's square water has not been noticed.
} 
comparison to nonpolar solvents, as well as in terms of the possible hydration effect.

The pure and mixture models are defined in the following section. Although presented previouly by Nadler and Krausche, square water is explained for clarity, and its connection with ferroeletric vertex models is discussed. Simulations and results are presented in Section III and a summary presented in the final section.

\section{The models and simulations}

Square water consists of a square lattice whose points are occupied by oxygens and whose lines ${ }^{2}$ are occupied by hydrogens. There are six different states per water particle, related to the possible distributions of two molecular hydrogens on four lattice lines (Fig. 1a). A hydrogen bond is present when the hydrogen atom of one molecule (donor) points to the oxygen of a neighbouring one (acceptor), and is absent when the molecules are both donors or both acceptors. Typical situations are illustrated in Fig. 1b. In the presence of a hydrogen bond (HB) an energy $-\epsilon$ is attributed to the lattice line, otherwise the energy is zero. Because the bond between a pair of neighbouring molecules depends on the relative position of the hydrogen atoms, the model mimics the directional nature of HBs [11], despite the fact that it is a lattice model. Hydrogen bonding is the only interaction considered, since van der Waals interactions which should also be present are an order of magnitude smaller than HBs $[12,18]$.

The model for water is a generalization of square ice [19, 20] (see Fig. 1c), through the inclusion of thermal fluctuations which produce rotations of the molecules. It must not be confused with ice rule ferroelectric or vertex models [21], for which the energy of neighbouring pairs is either zero or infinite (no broken bonds), and different energies are attributed to the particle states. In the present model particles are allowed six states, but the bond graph representation [21] of the vertices is no longer adequate. As in generalized ferroeletric models, the ice condition is broken, but in a completely different way: each oxygen atom is always accompanied by two hydrogens near it (representing a neutral molecule), but it is possible to have two, none or one hydrogen atom on each lattice line. A HB is present only in the last case. Therefore, unlike in vertex models, the energy depends on the neighbourhood. ${ }^{3}$

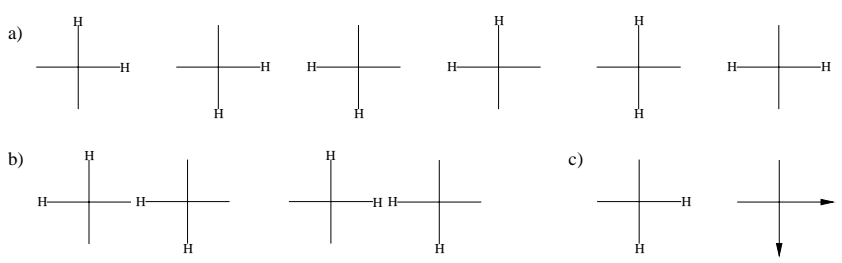

Figure 1. a) The six states for the water particle. Oxygens are on lattice sites. b) An HB is present if there is one hydrogen atom on the lattice line. The first pair contributes with HB energy $-\epsilon$. Rotation of the left molecule "breaks" the bond, as in the second pair. c) Vertex representation of water molecule.

The hydrophobic properties of a square water solvent are tested on a model for aqueous solution of apolar molecules. Single-site nonpolar particles are introduced on the lattice and, for simplicity, are considered inert (interactions with water or amongst themselves are disregarded). Thus HBs are allowed to "break" due either to the presence of nonpolar particles or, as in the pure water model, as a result of thermal fluctuations.

The properties of the two models were studied through Monte Carlo simulations in the canonical ensemble. Two types of movement are needed in order to go through the phase space of the system. For the pure system, a new state is obtained using a local movement of the HB network: a water molecule is randomly chosen and a new state selected. In the mixture system, with the concentration of nonpolar particles fixed, an additional movement is introduced which consists of a random distance exchange [22] between water and nonpolar particles (a global movement). In the latter case, the particles are randomly chosen and their spatial positions exchanged. The state of the water molecule in its new position is chosen randomly. In both cases the Metropolis probability is used [23]. Random numbers are generated from ran2 [24].

\section{Results and discussion}

As shown previously by Nadler and Krausche [16] from simulations, square water does not present a phase transition. In Fig. 2 we present similar results for several lattice sizes, as well as exact calculations for small lattices: there are no significant differences in the specific heats of lattice sizes greater than $\mathrm{L}=10 .^{4}$

\footnotetext{
${ }^{2}$ In order to maintain a clear distinction between lattice bonds and hydrogen bonds we have chosen to call the former lattice lines.

${ }^{3}$ The model could be written in terms of generalized Potts variables with directional rules for the interactions.

${ }^{4}$ This result could be interpreted qualitativaly due to the high entropy of the ground state (the entropy of the ice model is approximately $1.5 k_{B}[19]$ per particle, and therefore the competition between a state with low energy and another one with high entropy is reduced.)
} 


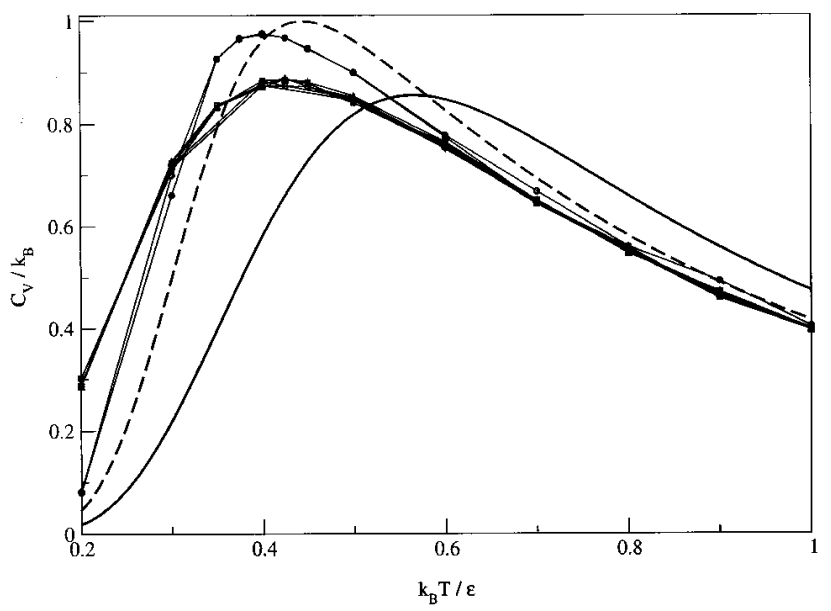

Figure 2. Specific heat for pure square water. Circles, triangles, squares and diamonds correspond to $L=4,10,15,30$ Monte Carlo results, respectively. Grey and black symbols represent ordered and disordered initial conditions. Continuous and dashed lines are exact results for $L=2,3$. Smooth behaviour as a function of system size indicates absence of phase transition.

The model solution was studied for concentration $20 \%$. The system exhibits phase separation below a reduced transition temperature $t \equiv k_{B} T / \epsilon \approx 0.4$ which is indicated by the peak in the specific heat shown in Fig. 3. Lattice gas data at $20 \%$ obtained from a random exchange algorithm [25] are shown for comparison, as well as the exact result [26] for the homogeneous phase.

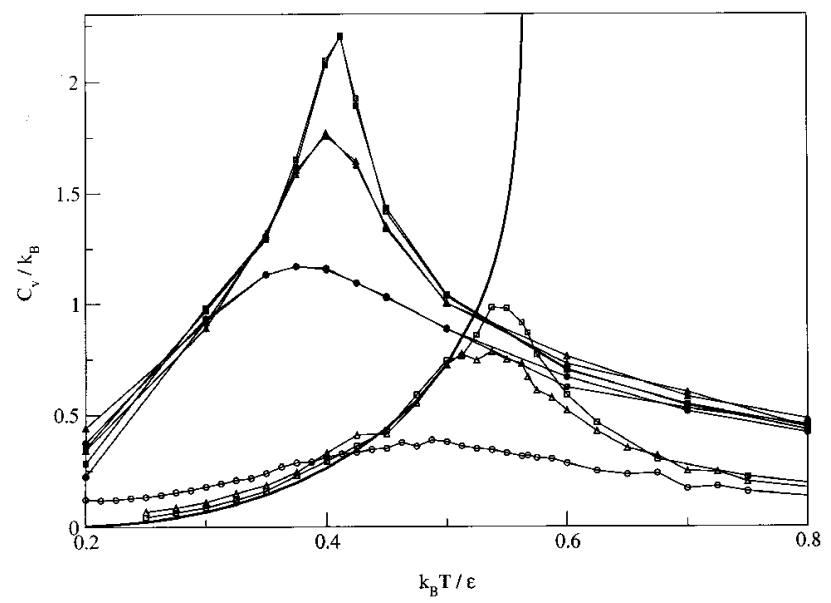

Figure 3. Specific heat (full symbols) for the solution of apolar particles at $20 \%$ concentration. Gray and black symbols represent ordered and disordered initial conditions. Lattice gas specific heat is shown for comparison (empty symbols for $\mathrm{MC}$ results and line for exact result). Circles, triangles and squares respectively for $L=10,30,60$. Peaks indicate phase separation at low temperature.

The square water and lattice gas mixtures may be compared respectively to associated[18], and van der Waals liquids acting as solvents. Associated liquids present a higher boiling temperature and lower solubility than corresponding van der Waals liquids. The difference between these two model solvents is the directionality of the HB interaction. Comparison of the specific heat peaks shows that phase separation occurs for a larger reduced transition temperature in the case of the lattice gas. In order to understand this result we must look at the absolute transition temperature. van der Waals bond energies $\epsilon(v d W)$ are usually an order of magnitude lower than HB energies $\epsilon(H B)[18]$. Thus

$$
\frac{T_{w}}{T_{v d w}} \approx 0.7 \frac{\epsilon_{H B}}{\epsilon_{v d w}} \approx 7
$$

The absolute transition temperature of the aqueous solution model $\left(T_{w}\right)$ is an order of magnitude greater than that of the van der Waals solution $\left(T_{v d W}\right)$, indicating lower solubility in the first case.

As to solubility properties of the model, it can be argued that it increases with temperature, following the tendency of the system to become homogeneous at higher temperatures[27]. It remains to be seen if this property persists at smaller overall concentrations.

The study of water structure was performed in terms of frequency of bonds. At ambient temperatures the liquid water net presents around $88 \%$ of maximum number of bonds [13]. In Fig. 4 we present a histogram of particles classified according to the number of bonds (there are between 0 and 4 HBs per particle) for pure square water typical of this region ( 80 and $92 \%$ of HB, the latter corresponding to a temperature slightly below transition).

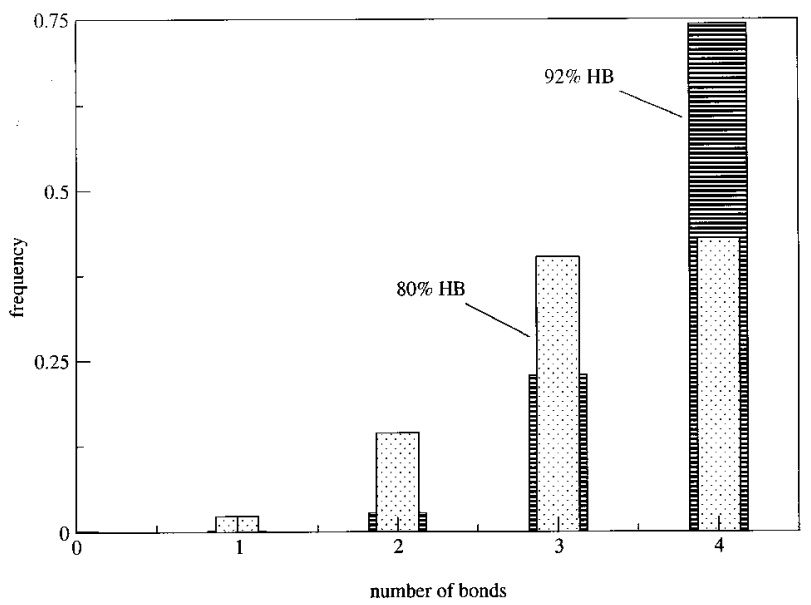

Figure 4. Pure square water: particles classified according to the number of bonds. $80 \%$ of bonds are present for reduced temperature $t=0.7$ and $92 \%$ of bonds for $t=0.4$. Monte Carlo data for $L=60$.

For the mixture we have classified separately first hydration shell (first neighbours of apolar particles) and bulk water particles. The water molecules around the nonpolar particles make fewer bonds and have a smaller frequency for maximum bond number. This result is 
in agreement with that obtained from simulations of atomic detail solvent [8]. On the other hand, frequency of maximum bond number in the first hydration shell shows a smaller dependence on temperature as compared to bulk water, as can be seen in Fig. 5. The hydration shell shows a slower rate of decay of bonds with temperature above, and especially below the phase separation transition. Thus the ratio of frequencies of maximum bond number for shell vs bulk water increases with temperature. Similar results have been seen in simulations of atomic detailed water[8, 9], albeit in contradiction to the simplified off-lattice MB model[28].

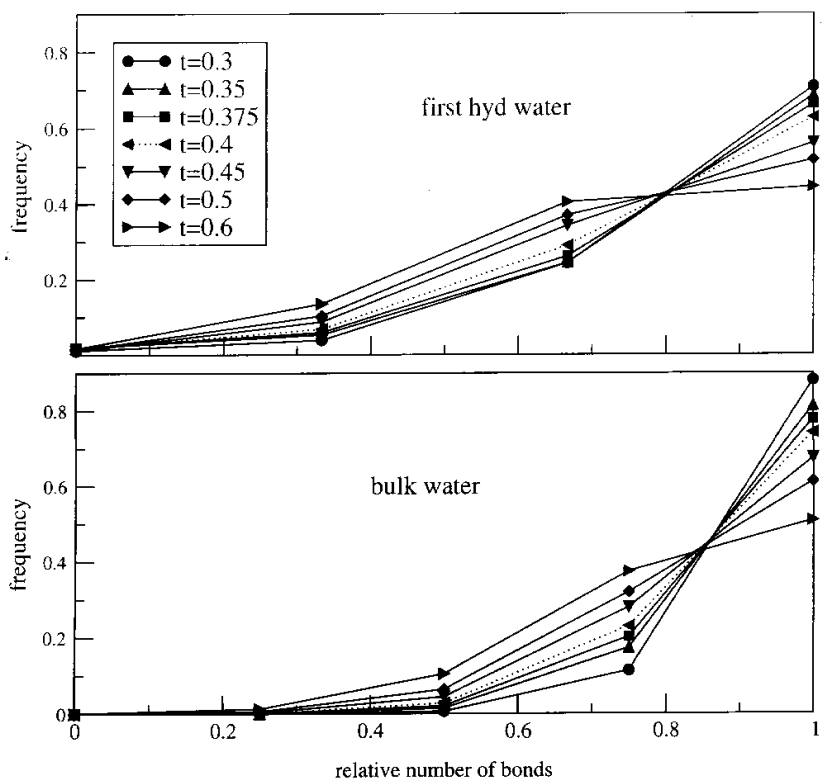

Figure 5. First hydration shell and bulk water particles classified according to the number of bonds. Dashed line is phase separation temperature. Note slower rate of variation with temperature for hydration shell particles both above and below the transition temperature. Monte Carlo data for $L=60$.

\section{Conclusions}

We have compared directional bonded (square water) and simple-bonded (lattice gas) solvents for apolar solutes in relation to phase-separation in terms of their specific heats. For $20 \%$ solute concentration, square water presents a lower reduced transition temperature, but the associated $\mathrm{HB}$ net which it is intended to describe presents a higher absolute transition temperature. At the concentration studied, solubility increases with temperature, contrary to what one would expect for apolar solutes in water. Simulations at different concentrations must be undertaken in order to check whether this is an overall property of the model.

Hydrophobic properties of square water were also studied in terms of frequency of bonds of bulk and hydration shell water. Hydration shell water "looses" structure with temperature at a slower rate than bulk water. This might contain some indication of the presence of "hydrophobic" hydration [1].

The study of the effect of such properties on aggregation or structure of amphiphilic molecules may be of interest.

\section{Acknowledgments}

We thank P. Wolf for pointing out the relation to the "spin ice" problem and C.E.I. Carneiro for discussions of simulations. NG acknowleges financial support from Fapesp.

\section{References}

[1] H. S. Frank and M. W. Evans, J. Chem. Phys. 13, 507 (1945).

[2] G. L. Pollack, Science 251, 1323 (1991).

[3] W. Luck; M. Blandauer and M. Fox in Water: a Comprehensive Treatise, ed F. Franks, Plenum 1973.

[4] K. Hallenga, J. R. Grigera and H. J. C. Berendsen, J. Phys. Chem. 84, 2381 (1980).

[5] See, for example, D. E. Smith and A. J. Haymet, J. Chem. Phys. 98, 6445 (1993); S. Ludemann, H. Schreiber, R. Abseher and O. Steinhauser, J. Chem. Phys. 104, 286 (1996).

[6] E. Fois, A. Gamba and C. Redaelli, J. Chem. Phys. 110, 1025 (1999).

[7] B. Madan and K. Sharp, Biophys. Chem. 78, 33 (1999).

[8] S. Urahata and S. Canuto, Chem. Phys. Lett. 313, 235 (1999).

[9] J. R. Grigera, S. G. Kalko and J. Fischbarg, Langmuir 12, 154 (1996); J. Phys. Chem. B102, 8941 (1998).

[10] G. T. Barkema and B. Widom, J. Chem. Phys. 113, 2349 (2000).

[11] F. H. Stillinger, Science 209, 451 (1980).

[12] D. Eisenbeg and W. Kauzmann, The structure and Properties of Water, Clarendon, 1969.

[13] W. L. Jorgensen, J. Chandrasekhar, J. D. Madura, R. W. Impey, M. L. Klein, J. Chem. Phys. 79, 926 (1983).

[14] H. J. C. Berendsen, J. R. Grigera and T. P. Straatsma, J. Phys. Chem. 91, 6269 (1987).

[15] See references in H. E. Stanley, S. V. Buldyrev, M. Canpolat, M. Meyer, O. Mishima, M. R. Sadr-Lahjany, A. Scala, F. W. Starr, Phys. A 257, 213 (98).

[16] W. Nadler and T. Krausche, Phys. Rev. A 44, R7888 (1991); T. Krausche and W. Nadler, Z. Phys. B, 86, 433 (1992).

[17] A. P. Ramirez, A. Hayashi, R. J. Cara, R. Siddharthan and B. B. Shastry, Nature 399, 333 (1999).

[18] P. A. Egelstaff, An Introduction to the Liquid State, Clarendon, 1999. 
[19] E. H. Lieb, Phys. Rev. Letters 18, 692 (1967).

[20] J. Nagle, J. Math. Phys. 7, 1484 (1966).

[21] E. H. Lieb and F.. Y. Wu, "Two-dimensional Ferroelectric Models," in Phase Transitions and Critical Phenomena, vol.1, (editors: C. Domb and M.S. Green) (1972).

[22] C. Shida and V. B. Henriques, IJMP C 11, 1 (2000).

[23] N. Metropolis, A. W. Rosenbluth, M. N. Rosenbluth, A. H. Teller, E. Teller, J. Chem. Phys. 21, 1087 (1953).
[24] W. H. Press, S. A. Teukolsky, W. T. Vetterling, and B. P. Flannery, Numerical Recipes in $C$, second edition, Cambrigde, 1998.

[25] C. Shida, PhD thesis, Universidade de São Paulo, 1998.

[26] L. Onsager, Phys. Rev. 65, 117 (1944).

[27] P. W. Atkins, Physical Chemistry, fourth edition, Oxford, 1993.

[28] K. A. T. Silverstein, A. D. J. Haymet and K. A. Dill, J. Chem. Phys. 111, 8000 (1999). 